\title{
SUBSTÂNCIAS HÚMICAS SOBRE QUALIDADE TECNOLÓGICA DE VARIEDADES DE CANA-DE-AÇÚCAR
}

\section{HUMIC SUBSTANCES ON TECHNOLOGICAL QUALITY OF SOME SUGARCANE VARIETIES}

\author{
Marina Munhoz ROSATO ${ }^{1}$ \\ Antonio César BOLONHEZI ${ }^{2}$ \\ Luiz Henrique Zuculo FERREIRA ${ }^{3}$
}

\begin{abstract}
RESUMO
A expansão da cultura canavieira está ocupando áreas com solos de cerrado, de baixa fertilidade e caracterizados como ambientes estressante. Assim, é necessário avaliar outras tecnologias com objetivo de se aumentar a produtividade e a quantidade de sacarose. Assim sendo, este trabalho teve como objetivo avaliar a influência das substâncias húmicas (SHs ácidos húmicos $-0,12 \mathrm{~kg} \mathrm{~kg}^{-1}$; ácidos fúlvicos $-0,03 \mathrm{~kg} \mathrm{~kg}^{-1}$ ) aplicadas no sulco de plantio da cana-de-açúcar e seus efeitos na qualidade tecnológica da cana. O experimento foi instalado na Fazenda Santa Terezinha, localizada no município de Pereira Barreto - SP, num delineamento experimental de blocos casualizados em esquema de parcelas subdivididas, contendo 16 tratamentos $8 \times 2$ e quatro repetições, tendo como tratamentos as seguintes variedades SP89-1115, SP813250, SP83-2847, SP91-3011, RB72454, RB867515, RB835054 e SP91-3440 tratadas e não tratadas com SHs. Os resultados permitem concluir que o efeito das substâncias húmicas $(\mathrm{SHs})$ sobre o acúmulo de sacarose foi positivo para a variedade SP91-3011; promoveram redução no teor de açúcares redutores nas variedades RB835054 e RB867515 em determinadas épocas da safra e, não afetaram as outras características tecnológicas da cana-de-açúcar.
\end{abstract}

Palavras-chave: Saccharum spp; ácidos húmicos e fúlvicos; teor de sacarose.

ABSTRACT
As the sugarcane crop is occupying great areas in savannah soils, many of these areas can be characterized as stressful environments, it becomes inevitable the use of available technologies for the increment in sucrose. This study aimed to evaluate the influence of humic substances ( $\mathrm{SHs}$ - humic acids $-0,12 \mathrm{~kg} \mathrm{~kg}^{-1}$; fulvic acids $-0,03 \mathrm{~kg} \mathrm{~kg}^{-1}$ ) applied in the planting furrow in the technological quality of the sugarcane. The experiment was conducted at Santa Terezinha Farm, located in Pereira Barreto, state São Paulo, Brazil. A randomized block design in a split plot scheme, containing 8 varieties: SP891115, SP81-3250, SP83-2847, SP91-3011, RB72454, RB867515, RB835054 and SP91-3440, treated or not with SHs, in four repetitions was used. The results showed that humic substances (SHs) were positive for variety SP91-3011 on sucrose accumulation; they promoted reduction in the glucose and fructose content of RB835054 and RB867515 varieties in certain times of the crop and, they did not affect the other technological characteristics of the sugarcane.

Key-words: Saccharum spp; humic and fulvic acids; sucrose content.

\footnotetext{
1Discente em Agronomia da Faculdade de Ilha Solteira (FEIS) / Universidade Estadual Paulista "Júlio de Mesquita Filho" (UNESP), Ilha Solteira, São Paulo, Brasil. E-mail: marinamrosato@ig.com.br

${ }^{2}$ Docente do Departamento de Fitotecnia, Tecnologia de Alimentos e Sócio - Economia - Faculdade de Ilha Solteira (FEIS) / Universidade Estadual Paulista "Júlio de Mesquita Filho" (UNESP), Av. Brasil n 56, Centro - C.P. 31 - Ilha Solteira, São Paulo, Brasil, CEP: 15385-000. Email: bolonha@agr.feis.unesp.br Autor para correspondência

${ }^{3}$ Engenheiro Agrônomo - Usina Santa Adélia - Pereira Barreto, São Paulo, Brasil. E-mail: Izferreira@usinasantaadelia.com.br
} 
ROSATO, M.M. et al. Substâncias húmicas sobre qualidade tecnológica...

\section{INTRODUÇÃO}

A expansão da cultura canavieira para as regiões do centro-oeste está ocupando áreas com solos degradados e de baixa fertilidade. Neste contexto, a busca por novas tecnologias é importante para o manejo da cana nestes ambientes de produção denominados estressantes, na busca por ganhos em produtividade de colmos e qualidade tecnológica.

O' Donnell (1973) mencionou a existência de grupos funcionais nas substâncias húmicas que, quando em baixas concentrações atuam como reguladores de crescimento e podem agir como protetores contra efeitos oxidativos nas plantas estressadas. Em cana-de-açúcar, o conteúdo de sacarose dos colmos é a principal variável que expressa a qualidade tecnológica da matéria prima para a produção de açúcar e etanol. Canellas et al. (2006) mencionaram que os ácidos húmicos na presença de ácidos orgânicos exsudados de raízes, liberam moléculas bioativas de grupos auxínicos, que ativam a $\mathrm{H}^{+}$- ATPase (bomba de $\mathrm{H}+$ ) que também possuem um papel importante no transporte de sacarose. Pinto \& Carvalho (2003) concluíram que a aplicação de ácidos húmicos e fúlvicos da leornadita aumentaram significativamente a produtividade da videira, sem, no entanto, afetar o Brix da uva.

A utilização de $\mathrm{SHs}$ em baixas doses aplicadas no sulco de plantio da cana-de-açúcar é uma tecnologia recente e que precisa ser melhor investigada. Como a aplicação das SHs é feita no momento da cobertura dos toletes, não há custo de aplicação, desta forma, pode ser uma boa opção técnica na busca de incrementos na produção de sacarose por área. Neste sentido, Bolonhezi et al. (2007a) e (2008), observaram que as SHs incrementaram a produção de colmos e melhoraram significativamente o pol\%cana (PC). Todavia, Bolonhezi et al. (2007b) observou que as substâncias húmicas não promoveram efeito significativo sobre o teor de sacarose de soqueiras de cana-de-açúcar, independente da forma de aplicação. Por sua vez, Gullo (2007) constatou que o uso de $350 \mathrm{dm}^{3} \mathrm{ha}^{-1}$ em cana-planta de um produto a base de ácido húmico, promoveu aumento significativo na produtividade de cana, quando comparado com a testemunha.

Neste aspecto, objetivou-se neste trabalho avaliar os efeitos das SHs originada da leornadita sobre a qualidade tecnológica de variedades de cana-de-açúcar.

\section{MATERIAL E MÉTODOS}

O experimento foi instalado numa área de cana-de-açúcar localizada na Fazenda Santa Teresinha no município de Pereira Barreto - SP $\left(51^{\circ} 01^{\prime} 39,49\right.$ "W; $20^{\circ} 35^{\prime} 48,74$ "S; $\left.393 \mathrm{~m}\right)$. O solo da área é classificado como Argissolo Vermelho Eutrófico.

Na Figura 1 apresenta-se a precipitação pluvial mensal e a distribuição de chuvas na área experimental durante o ciclo de cana planta.

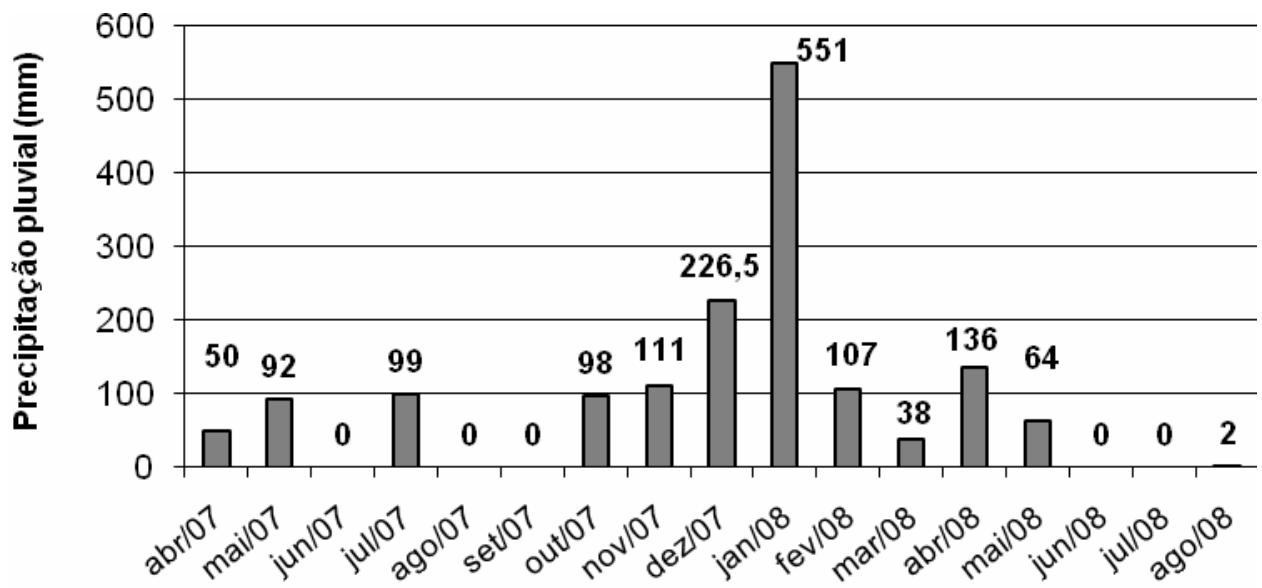

\section{Meses/Anos}

FIGURA 1 - Precipitação pluvial mensal $(\mathrm{mm})$ da área do experimento durante o ciclo da cultura.

O delineamento experimental utilizado foi o de blocos casualizados em esquema de parcelas subdivididas, contendo 16 tratamentos e quatro repetições, sendo 8 variedades: SP89-1115, SP813250, SP83-2847, SP91-3011, RB72454, RB867515, RB835054, SP91-3440 tratadas e não tratadas com substâncias húmicas (SHs). As parcelas foram constituídas de 5 linhas de $10 \mathrm{~m}$ de comprimento espaçadas de $1,50 \mathrm{~m}$.

As características do produto - substâncias húmicas (SHs) utilizado no experimento são: ácido húmico $-0,12 \mathrm{~kg} \mathrm{~kg}^{-1}$; ácidos fúlvicos $-0,03 \mathrm{~kg} \mathrm{~kg}^{-1}$; $0,08 \mathrm{~kg} \mathrm{~kg}^{-1}$ de nitrogênio e $0,04 \mathrm{~kg} \mathrm{~kg}^{-1}$ de potássio.

A sulcação foi realizada a $0,40 \mathrm{~m}$ de profundidade e a adubação utilizada no sulco de plantio foi de $620 \mathrm{~kg} \mathrm{ha}^{-1}$ da fórmula $\mathrm{N}-\mathrm{P}_{2} \mathrm{O}_{5}-\mathrm{K}_{2} \mathrm{O}$ 04-30-10.

O plantio foi feito no dia 23 de março de 2007 com mudas de 11 meses de idade, colocandose 12 gemas viáveis por metro de sulco. A 
ROSATO, M.M. et al. Substâncias húmicas sobre qualidade tecnológica...

aplicação das SHs foi feita durante a operação de cobertura dos toletes na dose de $20 \mathrm{dm}^{3} \mathrm{ha}^{-1}$, que foi realizada por implemento apropriado (cobridor), dotado de bicos para pulverizar sobre os toletes, uma calda de inseticida (fipronil: dose de $0,5 \mathrm{~kg} \mathrm{ha}^{-1}$ do p.c). Os herbicidas utilizados foram o clomazone na dose de $1,8 \mathrm{~kg} \mathrm{ha}^{-1}$ e diuron + hexazinone na dose de $1,5 \mathrm{~kg} \mathrm{ha}^{-1}$

Para as análises tecnológicas, foram colhidos 10 colmos por parcela sem broca e sem queima da palha. A análise tecnológica foi realizada no laboratório de pagamento da cana pelo teor de sacarose (PCTS) da Usina Santa Adélia - Pereira Barreto - SP. As variáveis analisadas foram: brix\% caldo, PC\%cana, fibra\%cana, AR\%cana e pureza, conforme métodos definidos pelo CONSECANA (2003).

\section{RESULTADOS E DISCUSSÃO}

As Figuras de $2 \mathrm{~A}$ a $2 \mathrm{H}$ representam as curvas de acúmulo de sacarose da cana planta das variedades avaliadas.Observando as curvas de acúmulo das variedades SP81-3250 (Figura 2A) e RB72454 (Figura 2B) constata-se que houve uma diferença significativamente maior no teor da pol da cana para o tratamento sem SHs nos dias 05/05/08 $(\mathrm{dms}=0,990)$ e 05/06/08 $(\mathrm{dms}=1,157)$, respectivamente. Mas, aparentemente verifica-se ainda uma inversão das tendências a partir do final do mês de julho. Ou seja, no início da fase nobre da safra de cana na região sudeste, as presenças de SHs mostram-se favoráveis ao acúmulo de sacarose para essas variedades.

Por outro lado, observa-se respostas positivas das SHs na variedade SP91-3011 (Figura 2C), apresentando uma maior porcentagem de sacarose nas avaliações feitas em junho e julho. Todavia, na variedade SP91-3440 (Figura 2D) observa-se que as SHs tiveram um efeito negativo no acúmulo de sacarose. Bolonhezi et al. (2008) mencionaram resultados positivos e expressivos das SHs sobre o teor de sacarose das variedades RB867515 e RB885028. Estes resultados são atraentes e merecem novas investigações, já que as tecnologias disponíveis para se aumentar o teor de sacarose na cana-de-açúcar se restringem ao uso de maturadores que exigem um alto nível tecnológico para a aplicação e são muito dependentes das condições climáticas. Além disso, verifica-se que as respostas variam muito de uma variedade para outra, indicando a necessidade de um manejo específico.

Analisando a Figura 2E, pode-se verificar que o acúmulo de sacarose da variedade SP901115 não sofreu influência das SHs. A variedade RB867515 (Figura 2F) mostrou-se insensível às substâncias húmicas. Por sua vez, a variedade SP86-2847 que é cortada em final de safra e é recomendada para ambientes estressantes, comportou-se como não responsiva às $\mathrm{SHs}$ para o acúmulo de sacarose (Figura 2G). Finalmente, analisando a Figura $2 \mathrm{H}$, nota-se que, durante $\mathrm{o}$ período de avaliação, as $\mathrm{SHs}$ promoveram efeito negativo sobre o pol\%cana na variedade precoce RB835054.

$\mathrm{Na}$ Tabela 1 pode-se observar que para a avaliação feita no início de maio as $\mathrm{SHs}$ reduziram significativamente a AR\%cana da variedade precoce RB835054 e na RB867515, mas aumentou na variedade de ciclo médio SP81-3250. No mês de junho pode-se observar também que houve redução significativa das SHs na AR\%cana somente para as variedades SP91-3011 e SP91-3440 consideradas de ciclo médio, ou seja, que devem ser colhidas nos meses de junho e julho. Finalmente, nota-se ainda que nos meses de julho e agosto, as SHs não afetaram o conteúdo de açúcares redutores das variedades avaliadas.

TABELA 1 - Valores médios de AR\%cana (porcentagens de açúcares redutores) de oito variedades de canade-açúcar com e sem SHs (substâncias húmicas) em quatro épocas de avaliação. Pereira Barreto, SP, 2008.

\begin{tabular}{|c|c|c|c|c|c|c|c|c|}
\hline \multirow{3}{*}{ Variedades } & \multicolumn{8}{|c|}{ AR\%cana } \\
\hline & \multicolumn{2}{|c|}{$5 / 5 / 2008$} & \multicolumn{2}{|c|}{$5 / 6 / 2008$} & \multicolumn{2}{|c|}{$5 / 7 / 2008$} & \multicolumn{2}{|c|}{$5 / 8 / 2008$} \\
\hline & Com SHs & Sem SHs & Com SHs & Sem SHs & Com SHs & Sem SHs & Com SHs & Sem SHs \\
\hline SP89-1115 & $0,86 a A B$ & $0,83 \mathrm{aA}$ & $0,54 a A$ & $0,53 a \mathrm{aB}$ & $0,56 a A$ & $0,42 \mathrm{aA}$ & $0,42 \mathrm{aA}$ & $0,42 \mathrm{aA}$ \\
\hline SP81-3250 & $1,11 \mathrm{aA}$ & $0,80 \mathrm{bA}$ & $0,63 a A$ & $0,52 \mathrm{aB}$ & $0,67 a A$ & $0,56 \mathrm{aA}$ & $0,61 \mathrm{aA}$ & $0,67 a A$ \\
\hline SP91-3011 & $0,96 a A B$ & $1,05 \mathrm{aA}$ & $0,62 a A$ & $0,79 \mathrm{bA}$ & $0,52 a A$ & $0,65 a A$ & $0,49 a A$ & $0,49 a A$ \\
\hline RB72454 & $0,78 \mathrm{aAB}$ & $0,75 \mathrm{aA}$ & $0,50 \mathrm{aA}$ & $0,51 \mathrm{aB}$ & $0,37 \mathrm{aA}$ & $0,40 \mathrm{aA}$ & $0,56 \mathrm{aA}$ & $0,49 \mathrm{aA}$ \\
\hline RB867515 & $0,70 \mathrm{aB}$ & $1,05 \mathrm{bA}$ & $0,49 a A$ & $0,62 a A B$ & $0,43 a A$ & $0,50 \mathrm{aA}$ & $0,42 \mathrm{aA}$ & $0,46 \mathrm{aA}$ \\
\hline SP83-2847 & $0,88 a A B$ & $0,73 a A$ & $0,53 a A$ & $0,54 a \mathrm{~B}$ & $0,55 a A$ & $0,50 \mathrm{aA}$ & $0,41 \mathrm{aA}$ & $0,47 a A$ \\
\hline RB835054 & $0,70 \mathrm{aB}$ & $0,97 \mathrm{bA}$ & $0,54 a A$ & $0,50 \mathrm{aB}$ & $0,46 a A$ & $0,40 \mathrm{aA}$ & $0,47 a A$ & $0,50 \mathrm{aA}$ \\
\hline SP91-3440 & $0,86 a A B$ & $0,87 \mathrm{aA}$ & $0,42 \mathrm{aA}$ & $0,60 \mathrm{bAB}$ & $0,44 \mathrm{aA}$ & $0,58 \mathrm{aA}$ & $0,62 \mathrm{aA}$ & $0,64 \mathrm{aA}$ \\
\hline DMS (variedades) & 0,388 & 0,388 & 0,220 & 0,220 & 0,455 & 0,455 & 0,300 & 0,300 \\
\hline DMS (tratamentos) & 0,246 & 0,246 & 0,140 & 0,140 & 0,290 & 0,290 & 0,190 & 0,190 \\
\hline CV (\%) & 8,83 & 8,83 & 15,49 & 15,49 & 27,69 & 27,69 & 20,74 & 20,74 \\
\hline
\end{tabular}

Médias seguidas de mesma letra não diferem entre si pelo teste de Tukey $(p<0,05)$. Coluna letra maiúscula e linha letra minúscula.

Scientia Agraria, Curitiba, v.11, n.1, p.043-048, Jan./Feb. 2010. 
ROSATO, M.M. et al. Substâncias húmicas sobre qualidade tecnológica...
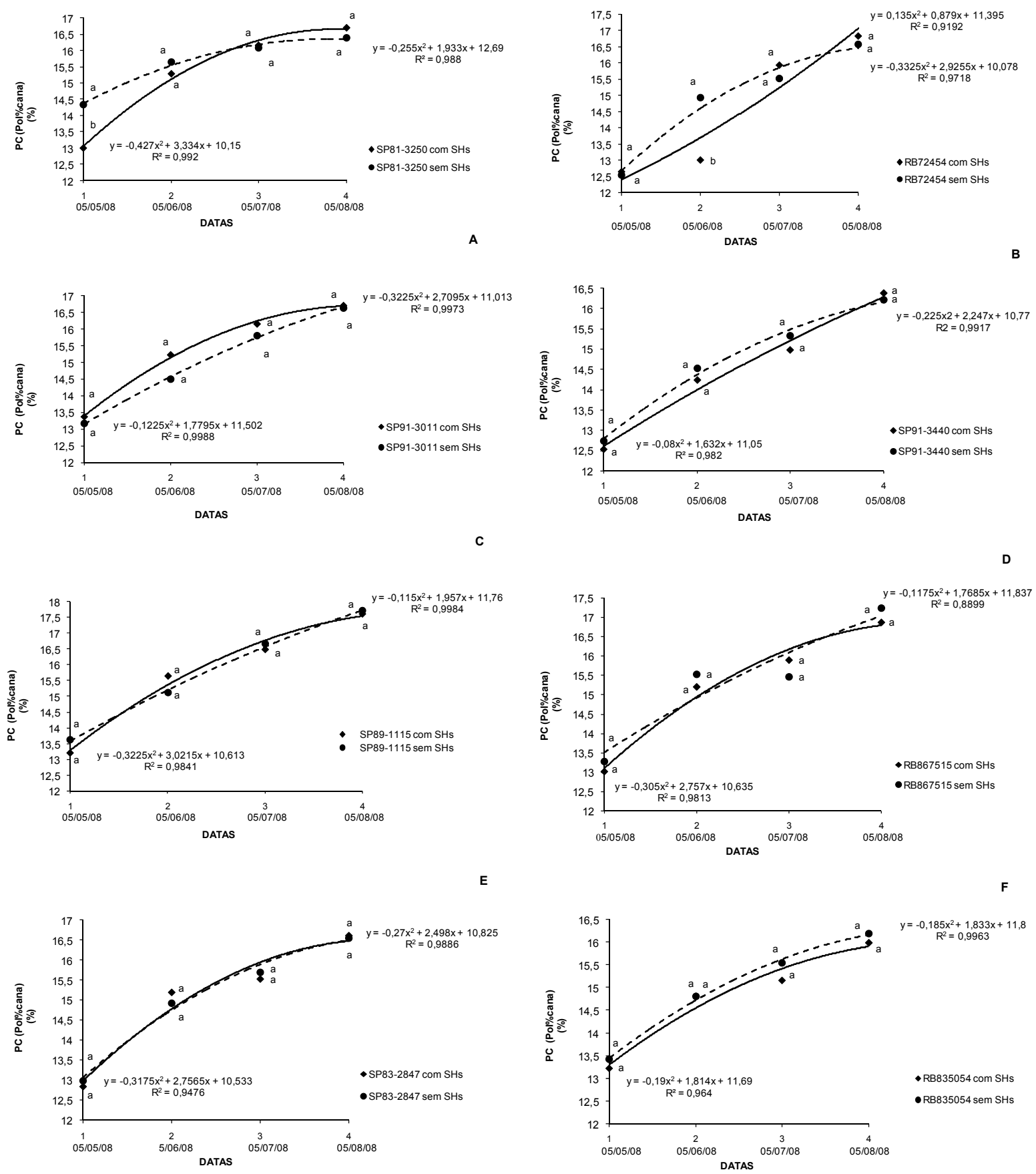

H

FIGURA 2 - Teor de sacarose (pol\%cana) das variedades de cana-de-açúcar SP81-3250 (A), RB72454 (B) SP91-3011 (C), SP91-3440 (D), SP89-1115 (E), RB867515 (F), SP83-2847 (G) e RB835054 (H) tratadas e não tratadas com SHs (substâncias húmicas) aplicadas no sulco de plantio. Pereira Barreto, SP, 2008. (Letras iguais não diferem entre si pelo teste de Tukey $(p<0,05)$ ). 
ROSATO, M.M. et al. Substâncias húmicas sobre qualidade tecnológica...

A porcentagem de fibra das variedades testadas não foi influenciada pelas SHs nos meses de safra avaliados (Tabela 2). Por outro lado, apenas na variedade RB72454 é que o Brix\%caldo foi significativamente maior no tratamento com as SHs no mês de agosto (Tabela 3).
Analisando os dados de pureza (Tabela 4), nota-se que apenas na primeira avaliação em maio, e somente a variedade SP81-3250 mostrou-se efeito negativo das $\mathrm{SH}$, como já se esperava, pois houve uma maior concentração de AR\%cana (Tabela 1).

TABELA 2 - Valores médios de fibra\%cana de oito variedades de cana-de-açúcar com e sem SHs (substâncias húmicas) em quatro épocas de avaliação. Pereira Barreto, SP, 2008.

\begin{tabular}{|c|c|c|c|c|c|c|c|c|}
\hline \multirow{3}{*}{ Variedades } & \multicolumn{8}{|c|}{ Fibra\%cana } \\
\hline & \multicolumn{2}{|c|}{$5 / 5 / 2008$} & \multicolumn{2}{|c|}{$5 / 6 / 2008$} & \multicolumn{2}{|c|}{$5 / 7 / 2008$} & \multicolumn{2}{|c|}{$5 / 8 / 2008$} \\
\hline & Com SHs & Sem SHs & Com SHs & Sem SHs & Com SHs & Sem SHs & Com SHs & Sem SHs \\
\hline SP89-1115 & $13,62 a A$ & $12,60 \mathrm{aA}$ & $12,82 \mathrm{aC}$ & $12,36 \mathrm{aC}$ & $12,77 \mathrm{aC}$ & $13,39 \mathrm{aC}$ & $13,41 \mathrm{aC}$ & $13,34 \mathrm{aC}$ \\
\hline SP81-3250 & $12,97 \mathrm{aA}$ & $12,40 \mathrm{aA}$ & $13,28 \mathrm{aBC}$ & $13,56 \mathrm{aAB}$ & $14,06 \mathrm{aAB}$ & $13,45 \mathrm{aC}$ & $14,56 \mathrm{aBC}$ & $14,24 \mathrm{aBC}$ \\
\hline SP91-3011 & $12,99 \mathrm{aA}$ & $13,70 \mathrm{aA}$ & $13,83 a A B C$ & $13,60 \mathrm{aAB}$ & $14,32 \mathrm{aAB}$ & $14,12 \mathrm{aB} C$ & $15,17 \mathrm{aAB}$ & $14,65 \mathrm{aABC}$ \\
\hline RB72454 & $13,36 \mathrm{aA}$ & $13,94 a A$ & $13,78 \mathrm{aABC}$ & $13,13 \mathrm{aBC}$ & $14,01 \mathrm{aB}$ & $14,12 \mathrm{aBC}$ & $14,86 a A B C$ & $15,02 \mathrm{aAB}$ \\
\hline RB867515 & $12,98 \mathrm{aA}$ & $12,82 \mathrm{aA}$ & $14,26 \mathrm{aAB}$ & $13,98 \mathrm{aAB}$ & $13,99 \mathrm{aB}$ & $14,09 \mathrm{aBC}$ & $15,20 a A B$ & $15,00 \mathrm{aAB}$ \\
\hline SP83-2847 & $14,30 \mathrm{aA}$ & $14,53 a A$ & $14,60 \mathrm{aA}$ & $14,67 a A$ & $15,19 a A$ & $15,43 a A$ & $16,13 a A$ & $15,77 \mathrm{aA}$ \\
\hline RB835054 & $15,40 \mathrm{aA}$ & $14,01 \mathrm{aA}$ & $14,16 \mathrm{aAB}$ & $14,22 \mathrm{aAB}$ & $14,34 \mathrm{aAB}$ & $14,29 a A B C$ & $15,10 \mathrm{aAB}$ & $15,04 a A B$ \\
\hline SP91-3440 & $13,12 \mathrm{aA}$ & $12,91 \mathrm{aA}$ & $13,89 \mathrm{aABC}$ & $13,98 \mathrm{aAB}$ & $14,29 \mathrm{aAB}$ & $14,72 \mathrm{aAB}$ & $14,87 \mathrm{aABC}$ & $14,55 \mathrm{aABC}$ \\
\hline DMS (variedades) & 2,530 & 2,530 & 1,145 & 1,145 & 1,162 & 1,162 & 1,504 & 1,504 \\
\hline DMS (tratamentos) & 1,600 & 1,600 & 0,725 & 0,725 & 0,734 & 0,734 & 0,952 & 0,952 \\
\hline $\mathrm{CV}(\%)$ & 8,09 & 8,09 & 3,60 & 3,60 & 4,13 & 4,13 & 4,26 & 4,26 \\
\hline
\end{tabular}

Médias seguidas de mesma letra não diferem entre si pelo teste de Tukey $(p<0,05)$. Coluna letra maiúscula e linha letra minúscula.

TABELA 3 - Valores médios de brix\%caldo de oito variedades de cana-de-açúcar com e sem SHs (substâncias húmicas) em quatro épocas de avaliação. Pereira Barreto, SP, 2008.

\begin{tabular}{|c|c|c|c|c|c|c|c|c|}
\hline \multirow{3}{*}{ Variedades } & \multicolumn{8}{|c|}{ Brix\%caldo } \\
\hline & \multicolumn{2}{|c|}{$5 / 5 / 2008$} & \multicolumn{2}{|c|}{$5 / 6 / 2008$} & \multicolumn{2}{|c|}{$5 / 7 / 2008$} & \multicolumn{2}{|c|}{$5 / 8 / 2008$} \\
\hline & Com SHs & Sem SHs & Com SHs & Sem SHs & Com SHs & Sem SHs & Com SHs & Sem SHs \\
\hline SP89-1115 & $18,67 \mathrm{aA}$ & $19,00 \mathrm{aA}$ & $20,72 a A B$ & $20,98 a A$ & $21,57 \mathrm{aA}$ & $21,93 a A$ & $23,65 \mathrm{aB}$ & $23,67 a A$ \\
\hline SP81-3250 & $19,40 \mathrm{aA}$ & $19,55 \mathrm{aA}$ & $20,57 a A B$ & $20,97 a A$ & $21,88 a A$ & $21,60 \mathrm{aA}$ & $23,05 \mathrm{aB}$ & $22,70 \mathrm{aA}$ \\
\hline SP91-3011 & $18,95 \mathrm{aA}$ & $19,82 \mathrm{aA}$ & $20,72 a A B$ & $19,94 \mathrm{aA}$ & $21,58 \mathrm{aA}$ & $21,65 a A$ & $23,37 \mathrm{aB}$ & $23,89 a A$ \\
\hline RB72454 & $18,22 \mathrm{aA}$ & $18,22 \mathrm{aA}$ & $19,20 \mathrm{aB}$ & $20,25 a A$ & $21,57 \mathrm{aA}$ & $21,34 a A$ & $26,09 \mathrm{aA}$ & $23,00 \mathrm{bA}$ \\
\hline RB867515 & $18,40 \mathrm{aA}$ & $18,80 \mathrm{aA}$ & $20,77 a A B$ & $21,05 a A$ & $21,48 a A$ & $21,07 a A$ & $23,84 \mathrm{aB}$ & $23,87 a A$ \\
\hline SP83-2847 & $18,32 \mathrm{aA}$ & $18,50 \mathrm{aA}$ & $20,72 a A B$ & $21,04 a A$ & $21,27 a A$ & $21,70 \mathrm{aA}$ & $23,15 \mathrm{aB}$ & $23,05 a A$ \\
\hline RB835054 & $19,35 \mathrm{aA}$ & $19,10 \mathrm{aA}$ & $21,21 \mathrm{aA}$ & $21,08 \mathrm{aA}$ & $20,60 \mathrm{aA}$ & $21,07 \mathrm{aA}$ & $22,91 \mathrm{aB}$ & $22,50 \mathrm{aA}$ \\
\hline SP91-3440 & $18,02 \mathrm{aA}$ & $18,17 \mathrm{aA}$ & $19,60 \mathrm{aAB}$ & $20,05 \mathrm{aA}$ & $20,62 \mathrm{aA}$ & $21,65 \mathrm{aA}$ & $22,95 \mathrm{aB}$ & $22,67 \mathrm{aA}$ \\
\hline DMS (variedades) & 1,955 & 1,955 & 1,809 & 1,809 & 1,680 & 1,680 & 1,956 & 1,956 \\
\hline DMS (tratamentos) & 1,240 & 1,240 & 1,111 & 1,111 & 1,060 & 1,060 & 1,240 & 1,240 \\
\hline CV (\%) & 1,40 & 1,40 & 6,36 & 6,36 & 4,18 & 4,18 & 3,24 & 3,24 \\
\hline
\end{tabular}

Médias seguidas de mesma letra não diferem entre si pelo teste de Tukey $(p<0,05)$. Coluna letra maiúscula e linha letra minúscula. 
ROSATO, M.M. et al. Substâncias húmicas sobre qualidade tecnológica...

TABELA 4 - Valores médios de pureza (\%) de oito variedades de cana-de-açúcar com e sem SHs (substâncias húmicas) em quatro épocas de avaliação. Pereira Barreto, SP, 2008.

\begin{tabular}{|c|c|c|c|c|c|c|c|c|}
\hline \multirow{3}{*}{ Variedades } & \multicolumn{8}{|c|}{ Pureza (\%) } \\
\hline & \multicolumn{2}{|c|}{$5 / 5 / 2008$} & \multicolumn{2}{|c|}{$5 / 6 / 2008$} & \multicolumn{2}{|c|}{$5 / 7 / 2008$} & \multicolumn{2}{|c|}{$5 / 8 / 2008$} \\
\hline & Com SHs & Sem SHs & Com SHs & Sem SHs & Com SHs & Sem SHs & Com SHs & Sem SHs \\
\hline SP89-1115 & $85,19 a A$ & $85,58 a A B$ & $90,02 \mathrm{aA}$ & $89,15 a A$ & $91,63 a A$ & $92,11 \mathrm{aA}$ & $90,26 a A B$ & $90,42 a A$ \\
\hline SP81-3250 & $84,94 a A$ & $87,41 \mathrm{bA}$ & $89,72 \mathrm{aAB}$ & $89,89 a A$ & $90,40 \mathrm{aA}$ & $90,16 a A B$ & $89,59 a A B$ & $88,66 a A$ \\
\hline SP91-3011 & $84,79 a A$ & $84,77 \mathrm{aAB}$ & $89,60 a A B$ & $88,41 \mathrm{aA}$ & $92,13 a A$ & $89,48 \mathrm{bAB}$ & $89,31 \mathrm{aAB}$ & $89,33 a A$ \\
\hline RB72454 & $84,24 a A$ & $84,23 \mathrm{aAB}$ & $86,97 \mathrm{aB}$ & $88,79 a A$ & $90,28 \mathrm{aA}$ & $89,47 a A B$ & $89,62 a A B$ & $89,77 a A$ \\
\hline RB867515 & $86,31 \mathrm{aA}$ & $84,65 a A B$ & $89,84 a A B$ & $90,12 \mathrm{aA}$ & $90,44 a A$ & $89,84 a A B$ & $90,71 \mathrm{aAB}$ & $89,90 \mathrm{aA}$ \\
\hline SP83-2847 & $86,08 \mathrm{aA}$ & $86,76 a A B$ & $90,65 a A$ & $90,36 \mathrm{aA}$ & $90,80 \mathrm{aA}$ & $90,70 \mathrm{aAB}$ & $91,20 \mathrm{aA}$ & $90,67 a A$ \\
\hline RB835054 & $85,46 \mathrm{aA}$ & $85,96 a A B$ & $90,13 \mathrm{aA}$ & $89,54 a A$ & $90,30 a A$ & $90,67 a A B$ & $89,32 a A B$ & $89,65 a A$ \\
\hline SP91-3440 & $83,91 \mathrm{aA}$ & $84,06 \mathrm{aB}$ & $88,66 \mathrm{aAB}$ & $88,53 a A$ & $89,26 \mathrm{aA}$ & $87,77 \mathrm{aB}$ & $88,64 a \mathrm{a}$ & $88,34 a A$ \\
\hline DMS (variedades) & 3,231 & 3,231 & 2,912 & 2,912 & 3,670 & 3,670 & 2,517 & 2,517 \\
\hline DMS (tratamentos) & 2,047 & 2,047 & 1,844 & 1,844 & 2,316 & 2,316 & 1,595 & 1,595 \\
\hline CV (\%) & 1,34 & 1,34 & 1,37 & 1,37 & 2,16 & 2,16 & 1,06 & 1,06 \\
\hline
\end{tabular}

Médias seguidas de mesma letra não diferem entre si pelo teste de Tukey $(p<0,05)$. Coluna letra maiúscula e linha letra minúscula.

\section{CONCLUSÕES}

De acordo com os resultados obtidos e considerando as condições e o período de realização do experimento, pode-se concluir: i) O efeito das SHs sobre o acúmulo de sacarose foi positivo para a variedade SP91-3011; ii) As SHs promovem no mês de maio redução no teor de açúcares redutores das variedades RB867515 e RB835054; iii) As SHs não afetaram as outras características tecnológicas da cana-de-açúcar; iiii) As SHs podem ser uma técnica importante para a melhoria da qualidade da matéria prima e para o manejo varietal.

\section{REFERÊNCIAS}

1. BOLONHEZI, A. C. et al. Substâncias húmicas aplicadas no sulco de plantio de duas variedades de cana-de-açúcar. In: ENCONTRO BRASILEIRO DE SUBSTÂNCIAS HÚMICAS - EBSH, 7., 2007, Florianópolis. Resumos... Florianópolis: UFSC, 2007a. p. 70

2. BOLONHEZI, A. C. et al. Modos de aplicação de substâncias húmicas em soqueira de cana-de-açúcar. In: ENCONTRO BRASILEIRO DE SUBSTÂNCIAS HÚMICAS - EBSH, 7., 2007, Florianópolis. Resumos... Florianópolis: UFSC, 2007b. p. 71.

3. BOLONHEZI, A. C. et al. Ácidos húmicos e fúlvicos aplicados no sulco de plantio de cana-de-açúcar em solo de cerrado. In: CONGRESSO NACIONAL DA STAB, 9., 2008, Maceió. Anais... Maceió: STAB/Gráfica, 2008. p. 559-564.

4. CANELLAS, L. P. et al. Efeitos fisiológicos de substâncias húmicas - o estímulo às H+-ATPases. In: FERNANDES, M. S. (Ed.). Nutrição mineral de plantas. Viçosa: Sociedade Brasileira de Ciência do Solo, 2006. p.175-200.

5. CONSELHO DOS PRODUTORES DE CANA-DE-AÇÚCAR, AÇÚCAR E ÁLCOOL DO ESTADO DE SÃO PAULO (CONSECANA). Manual de Instruções. 4. ed. Piracicaba: Opinião, 2003. 116 p.

6. GULLO, M. J. M. Uso de condicionadores de solo a base de ácido húmico na cultura de cana-de-açúcar (Saccharum spp.). 2007. 59 p. Dissertação (Mestrado em Agronomia - Àrea de concentração: Fitotecnia) - Curso de Pós-Graduação em Agronomia, Escola Superior de Agricultura Luiz de Queiroz, Universidade de São Paulo, Piracicaba, 2007.

7. O' DONNELL, R. W. The auxin-like effects of humic preparations from leornadite. Soil Science, v. 116, n. 2, p. 106-112, 1973.

8. PINTO, P. A. da C.; CARVALHO, A.S. Eficiência agronômica de ácidos húmicos e fúlvicos da leonardita aplicados na cultura da videira Itália. In: SOLO ALICERCE DOS SISTEMAS DE PRODUÇÃO, 2003, Ribeirão Preto. Resumos... Ribeirão Preto: Agromídia Ltda, 2003. p. 1-4. 